\title{
Nanobots in Medical Field: A Critical Overview
}

\author{
Sorna Mugi Viswanathan ${ }^{1}$ \\ Department of Electronics and Communication \\ Engineering KGiSL Institute of Technology, \\ Coimbatore,India
}

\author{
Anitha. $S^{2}$ \\ Department of Electronics and Communication \\ Engineering KGiSL Institute of Technology, \\ Coimbatore,India
}

\author{
Revanth Rajan ${ }^{3}$ \\ Department of Electronics and Communication Engineering \\ KGiSL Institute of Technology, Coimbatore, \\ India
}

\begin{abstract}
Today, the health care industry is focusing on developing minimum invasive techniques for diagnosis, as well as for the treatment of ailments. Advancement in technology is more essential for the treatment of many problems such as implant of bones and membranes. One such technique flourishes robots using nanotechnology which are known as nanobots. Nano robotics is an emerging technology creating machines or robots whose components are at or near the scale of a nanometre $\left(10^{-y}\right.$ meters). Nanobots will help to build a platform between the technological gaps of physics, chemistry and biology on the nano-scale. These nanobots can address the path to many creative approaches and will result in new methods and products for both technological and medical-pharmaceutical applications. Furthermore, nanobots are good applicants for complex treatments with their size being very small. Nanobots are used as drug delivery systems and contrast agents. It is contended that coated nanobots are functionalized with targeted molecules are interacting with external devices and offering real potentials for medical applications. This paper directs how a nanobot work and how it contribute to medical robotization with their advantages and disadvantages.
\end{abstract}

Keywords - Nanotechnology, nanobots, technological gap, drug delivery

\section{INTRODUCTION}

Nanobots are robots that carry out a very specific function and are approximately 50-100 $\mathrm{nm}$ wide. They can be used very actively for drug delivery. Normally, drugs pass through the entire body before they reach the diseaseaffected area. Using nanotechnology, the drug can be picked out to a specific site which can reduce the chances of possible side effects. More specifically, Nano robotics refers to the nanotechnology engineering discipline of designing and building Nano robots ranging in size from $0.1-10$ micrometers and is constructed in a nanoscale or molecular components. In the present circumstances, the nano devices which are under research and development are known by the terms nanobots, nanoid, nanite, nanomachine or nanomite. Nanomachines are used primarily in the research and development phase but some primitive molecular machines and nanomotors have been tested.

The study of manipulating matter on an atomic and molecular scale based on nanotechnology is basically termed as nanobots. Generally the size of nanobots lies between 1 to $100 \mathrm{~nm}$. It can play a major role in medical applications, especially for targeted interventions into the human body through the vascular network. The first crucial step toward developing real-world applications for targeted drug delivery and other uses are represented by recent fabrication, actuation, and steering demonstrations of nanoscale robots. It has huge impact in targeted cancer therapy, such as delivering therapeutic agents directly to the tumor through the vascular network. Today, when we talk about nanobots, the self-propelled nanomotors and other biodegradable nano devices made of bio-nano components, which carry cargo to the target sites, i.e. deliver drugs to diseased cells. For example, these nanorobots can transport molecular payloads throughout the body by programming and cause on-site tumor blood supply blockages which can lead to tissue death and thus shrink the tumor. Currently nanobots and their application in the medical field are under development. In association with medicine, nanobots are programmed to perform specific biological tasks and when they are injected into the blood, they work on cancer cells or any other affected cells. Nanobots blended with biological research will set a new milestone in the development of medical studies.

\section{ADVANTAGES OF NANOROBOTICS OVER CONVENTIONAL MEDICAL TECHNIQUES}

We Homo sapiens (advanced humans) have always been fascinated with our own anatomy. Ages ago, techniques to diagnose body ailments as well as to repair them have been developed. The progression of humanity has been quite a lot in terms of safety and reliability of the procedure. Techniques such as endoscopy have been developed to give a better understanding of the innermost parts as well as aid diagnosis. But as we all know, all technology certainly has to be phased out sometime. And as historical procedures have developed to overcome the drawbacks of their predecessors, nanorobotics will aim to overcome the following drawbacks of today's medical technology:

1. Cut harmful tissue layers which take time to heal.

2. Painful Anesthesia can be used to limit the pain to a great extent, yet it is only for a short time.

3. Still there is no $100 \%$ success for delicate surgeries such as eye surgery. 
4. In any of the invasive techniques, the patient's life is totally in the hands of the operator, surgeon or physician. It is risky, as one mistake could take the life of the patient.

For last few centuries, conventional techniques of investigation and diagnosis have been widely used and thus, soon it is going to fall behind as the technological age advances. Also all these procedures will soon become robotically controlled by machines.

However scientists and researchers are working on compelling, reliable and bio-compatible approach. Instead of curing a disease from outside, they course to defend it inside the body. That is where medical nanorobotics comes in. The major advantages of this technology are:

1. Minimal or no tissue trauma.

2. Considerably less recovery time.

3. Less post-treatment care required.

4. Rapid response to a sudden change.

5. Continuous monitoring and diagnosis from

the inside.

Some features of nanobots would also allow us to store and process previous data, identify patterns and hence, help to predict the attack of an ailment. Nanobots can be guided externally as per programmed and deliver payloads such as drugs, or healthy cells to the specific location in the body. An added advantage is that these nanobots can navigate through natural biological pathways.

\section{NANOBOTS IN MEDICINE}

\section{A. Sugar level monitering bots}

The sugar level in the blood can be monitored by inserting special sensor nanobots into the blood in which an electrical impulse signal is emitted by microchips which are coated with human molecules. The drug carriers consist of walls that are just 5-10 atoms thick and inner drug-filled cell which is usually $50-100 \mathrm{~nm}$ wide. When they detect signs of the disease, thin wires in their walls emit an electrical pulse which causes the walls to melt and the drug to be released.

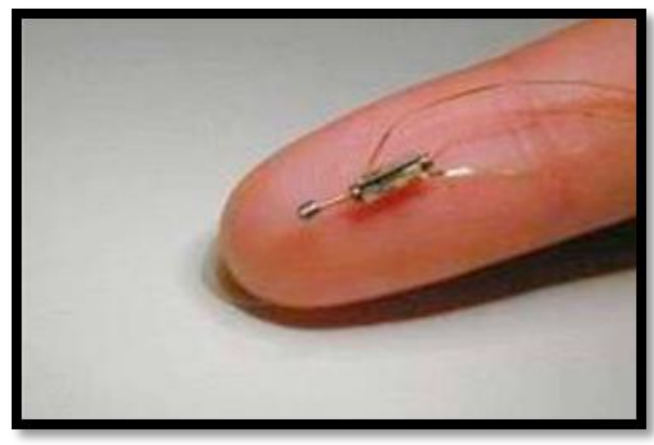

Fig:1. Device using nanobots for checking blood contents

An intensive advantage of using nanobots for drug delivery is that the the electrical pulse can be controlled which easily controls the amount and time of drug release to the specific site. Moreover, the walls melt and dissolve easily and are therefore harmless to the body.

\section{B. Enzyme-proprelled nanorobot}

Urea-coated nanotubes turn into a propulsion system in a urea-containing liquid because the enzyme breaks down the urea into gaseous products. A current in the liquid is generated by the reaction products since the tubes always have small asymmetries. This active motor based drug delivery approach promises an effective and improved drug delivery compared to conventional methods.

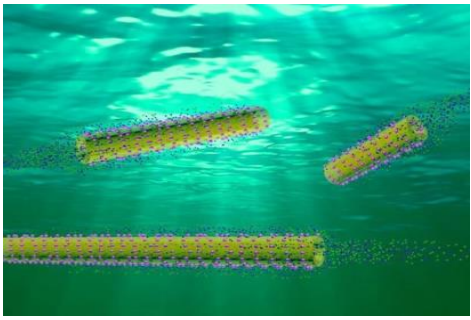

Fig: 2. Enzyme-propelled nanorobot

These nanobots show excellent acid-driven, self-propulsive properties with high cargo-loading capacities

\section{Cancer detection and treatment}

Nanorobots are successfully programmed by the scientists from Arizona State University and China's National Centre for Nanoscience and Technology (NCNT) to detect and shrink cancer tumours in the brain. With 25 million nanometers per inch, these miniature robots may provide an extra help that oncologists need to reduce cancer, such as enhancing their capabilities to detect, diagnose and treat cancer cells.

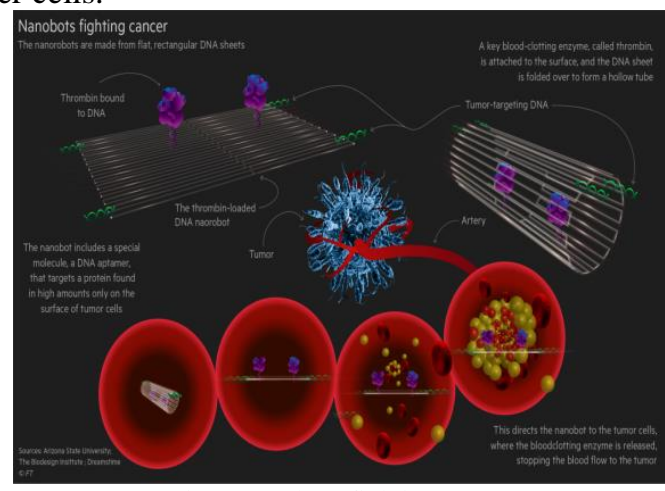

Fig3: Nanobots fighting cancer

Today, drug delivery for cancer is difficult to control. Chemotherapy anguishes healthy tissue in addition to malignant tissue. We cannot prevent the harm effects of chemotherapy on other parts of our body. But this is not done by nanobots. Nanobots could be used to deliver drugs particularly only to the tumor cells, thus preventing the auxiliary effect of the drug. Primarily, nanobots are sent to the targeted tissue or tumor to provoke it which is a part of machine gun approach but a lot of the bots will be wasted. However, only the tumor is provoked and not any other tissue in the entire body is affected. Now, a second wave of bots is sent to targeted tissue and this wave of bots contains the actual chemotherapy drug. It releases its payload i.e. the drug only after sensing the provoked tissue. Thus, we have highly concentrated targeted action, with no peripheral impact. 


\section{POTENTIAL USES OF NANOBOTS}

The budding uses for nanorobotics in medicine include prior diagnosis and targeted drug-delivery for cancer, surgery, pharmacokinetics, monitoring of diabetes and biomedical instrumentation. In such a deal, future medical nanotechnology is expected to make use of nanorobots that are injected into the patient's body to perform its work at a cellular level. These nanorobots should not identical because duplication leads to increase in device complexity, reduce its accuracy and tamper with the medical mission.

Nanotechnology provides a wide range of new technologies for developing adapted means to adjust the delivery of pharmaceutical drugs. Today, harmful side effects of treatments such as chemotherapy are mutually a result of drug delivery methods that doesn't distinguish their intended target cells accurately.

Another useful application of nanorobots is cooperating in the repair of tissue cells along with white blood cells. Mobilizing inflammatory cells or white blood cells (which include neutrophil granulocytes, lymphocytes, monocytes, and mast cells) to the affected area is the first response of tissues to any injury. Because of their small size, nanorobots could attach themselves to the surface of mobilized white blood cells to crunch their way out through the walls of blood vessels and arrive at the injury site, where they can cooperate in the tissue repair process.

\section{WAYS IN WHICH NANOBOTS CHANGES THE FUTURE}

\section{A. Detect Bacteria}

A lot of nanobot's estimate uses are related to medicine in some manner. For instance, it is believed that nanobots will be able to detect the presence of bacteria and other microbes in the human body, which in turn, means that they will be able to detect whether someone has been infected or not as well as what kind of response should be set based on the kind of infection.

\section{B. Detect Cancer}

It is esteemed that nanobots will be able to act as an early warning system, which will pick up changes in the human body that signal the mutation of healthy cells into cancer cells. By doing so, they will allow the implementation of solutions in a timely manner.

\section{Determines the Effectiveness of Drug}

One of the biggest challenges in medicine is to figure out the effect that a particular medicine is having on the patient so that the medical expounder can tackle the problem by decreasing the side effects. Moreover, determining the effectiveness of medicine is also important because it allows the medical expounder to know how to treat the patient as soon as possible. Nanobots will be able to help with both of these tasks.

\section{Detect Particular Chemicals}

On a associated note, it is believed that nanobots will be able to detect the presence of particular chemicals in the human body as well, which will provide crucial information to medical expounders about the condition of the patient so that it can be used to ensure more efficient and effective treatment.

\section{E. Deliver Cancer-Fighting Drugs}

Chemotherapy can be cruel on a cancer patient because it can kill cancer cells as well as the healthy cells surrounding the cancer cells. There has been some motion made regarding the use of nanobots to make sure that the cancer-fighting drugs are delivered right to the cancer cells, thus restricting the parallel damages. This means that nanobots can be used to target harder-to-reach portions of cancerous tumors, which in turn, means that there will be a potential increase in the chances of successful chemotherapy as well.

\section{F. Clear Blocked Blood Vessels}

There is a lot of interest coming up with potential solutions as well as potential preventatives for cardiovascular disease which is one of the most common killers. Theoretically, blockages in blood vessels which are responsible for both strokes and heart attacks can be cleared by using nanobots. But practically, if these bots are not able to wholly solve the problem, they are able to reduce the chances of dying from either one of those conditions, which will be an incredible improvement even.

\section{G. Serve as Antibodies}

Nanobots are used to boost the existing antibodies for the people with weak immune systems who cannot manage all the bacteria and other microbes that they await to attack. Here, the nanobots actually being used to potentially destroy the dangerous foreign substances in the human body. Alternatively, this could consist of the nanobots that direct the existing immune processes at the sources of danger.

\section{H. Clean Up Pollution}

In future, it might be possible to use nanobots to clean up pollutions, thus restoring polluted environments to a clean and virgin condition. Inspecting the impacts that pollution can cause the health of entire ecosystems including human health, nanobots can be considered as an inestimable boon because nanobots would be deployable in toxic sites thus reducing the risk to human counterparts.

\section{ADVANTAGES AND DISADVANTAGES OF NANOBOTS ADVANTAGES}

The main advantages of the bots are its speed and longevity. There are abundant benefits through nanobots that are provided by present methods of drug delivery. Nanobots are very specific and accurate with fewer side effects which release drugs in a controlled manner. It also minimizes surgeon mistakes. Computer controlled drug delivery and greater speed of drug action are its sublimit.

\section{DISADVANTAGES}

The main disadvantage is that it is expensive to design the nanobots and a lot of complications are involved in designing them as well. The most daunting obstacle is the power supply. For the bots to overcome the body's immune response, more research work has to be done so that. If the 
nanobots are misused by terrorists, it could even be used as bio-weapons and may become a threat to the society. If nanobacterias are present in our body, they can cause a serious of effects, which means nanobots are foreign to the body. Due to so many foreign particles inside the body, biodegradability will be a significant problem. Hence, a vigorous care has to be taken to overcome all these drawbacks. A harmful version of the bots could be created if nanobots self-replicate and our immune system can be challenged if we depend a lot on nanotechnology.

\section{CONCLUSION}

In the field of medicine, the use of nanorobotics has a wider scope than any other sub-field that has emerged to date. These bots can be used comparatively high anywhere in association with human physiology. It provides enormous advantages over conventional medicine such as lower cost, quicker recovery and low or almost no invasion. There will be a great revolution in medicine, comparable to the industrial revolution which reshapes the world in the age of integrative activity. With a flock of nanobots protecting us from inside, we could actually be free from disease in the next few decades with life expectancy. Cancer detection, data storage, and pipeline monitoring offers some of the strongest cases of development using nanorobotics for the future. Thus, nanorobotics is an ideal field to explore progressively.

\section{REFERENCES}

[1] “A REVIEW ON DNA NANOBOTS - A NEW TECHNIQUE FOR CANCER TREATMENT”. DEVASENA UMAI R, BRINDHA DEVI P*, THIRUCHELVI R Department of BioEngineering, School of Engineering, Veils Institute of Science, Technology and Advanced Studies, Pallavaram, Chennai- 600 117, Tamil Nadu, India. Email: pbrindhadevi@gmail.com.

[2] "NANOBOTS: THE FUTURE OF MEDICINE", Bhat A.S. Department of Instrumentation and Control, College Of Engineering, Pune (COEP), Shivajinagar, Pune-411005, Maharashtra, India.

[3] "Nanobots: The Future Of Medical Treatments", Somanna M B, INTERNATIONAL JOURNAL OF SCIENTIFIC \& TECHNOLOGY RESEARCH VOLUME 4, ISSUE 12, DECEMBER 2015 ISSN 2277-8616.

[4] Smart Nanobots: The Future in Nanomedicine and Biotherapeutics Usama Ahmad1 and Md Faiyazuddin2* 1 Faculty of Pharmacy, Integral University, Kursi Road, Lucknow, India2260262 Department of Pharmaceutics, College of Pharmacy, University of Hail, Post Box No. 2440, Hail City, KSA, Saudi Arabia.

[5] "Pharmacytes: An Ideal Vehicle for Targeted Drug Delivery Robert”, A. Freitas, Jr. Institute for Molecular Manufacturing, 555 Bryant Street, Suite 354, Palo Alto, California 94301, USA.

[6] "Nanomedicine: destination or journey?" C A Haberzettl TechnoMed Strategic Partners, Inc.,1988 Coventryville Rd, Pottstown, PA 19465, USA. Received 19 June 2002 Published 4 July 2002 Online at stacks.iop.org/Nano/13/R9.

[7] "Nanorobot Communication Techniques: A Comprehensive Tutorial", Adriano Cavalcanti Tad Hogg Bijan Shirinzadeh Hwee C. Liaw. CAN Center for Automation in Nanobiotech Sao Paulo, SP 01540, Brazil email: adrianocavalcanti@canbiotechnems.com HP Labs Palo Alto, CA 94304, USA email: tad_hogg@hp.com Robotics and Mechatronics Research Lab., Dept. of Mechanical Eng., Monash University Clayton, Melbourne, VIC 3800, Australia emails: \{bijan.shirinzadeh, hweechoo.liaw\}@eng.monash.edu.
[8] "Bioorthogonal catalysis: Rise of the nanobots", Article in Nature Chemistry • June 2015 DOI: 10.1038/nchem.2291 • Source: PubMed, CITATIONS 10, READS 379; author: Asier Unciti-Broceta The University of Edinburgh 79 PUBLICATIONS 973 CITATIONS.

[9] "Nanorobotics" - Wikipedia, From Wikipedia, the free encyclopaedia, https://en.wikipedia.org/wiki/Nanorobotics. 